\title{
G20 in Hamburg
}

Politik, Unvernehmen, Ausnahmezustand und das Ende der Postdemokratie

\author{
Daniel Mullis
}

\begin{abstract}
In Hamburg trafen sich am 7. und 8. Juli 2017 die G20. Die Tage waren von vielfältigen und massenhaften Protesten geprägt, die in Teilen heftig eskalierten. Im Artikel werden fünf Aspekte herausgearbeitet, die an sich nicht grundlegend neu sind, in der Summe aber für eine Zuspitzung politischer Auseinandersetzungen in Deutschland stehen und die G20-Tage zu einem wichtigen Ereignis machen: (1) eine deutliche Polarisierung politischer Positionen, (2) die Ausweitung antagonistischer Praxen, (3) die Suspendierung von Grundrechten, (4) mediale Eskalation und (5) die Militarisierung politischer Konflikte. Geleitet wird die Diskussion einerseits von Jacques Rancière und dessen Überlegungen zu Politik und Unvernehmen sowie von den Arbeiten zum Ausnahmezustand von Giorgio Agamben. Andererseits baut der Text auf eine qualitative Auswertung der medialen Berichterstattung um die G20 Proteste sowie auf Protestaufrufe und -bewertungen. Vor diesem theoretischen Hintergrund argumentiere ich, dass die fünf Aspekte in der Summe als Indikator für das Ende der Postdemokratie zu lesen sind.
\end{abstract}

Ersteinreichung: 28. August 2017; Veröffentlichung online: 20. Mai 2018

An english abstract can be found at the end of the document.

\section{Einleitung}

Am 7. und 8. Juli 2017 trafen sich die G20 in Hamburg. [1][2] Es war ein turbulenter Gipfel. Konflikte zwischen den Staats- und Regierungschef_innen traten zu Tage und das Treffen wurde von heftigen Protesten begleitet. Vom 1. bis zum 9. Juli 2017 beteiligten sich Zehntausende an vielfältigen Protestformen: von Yoga gegen G2o über einen Gegengipfel, Tanz- und Großdemonstrationen bis hin zu Aktionen des zivilen Ungehorsams und einer Parkbesetzung. Darüber hinaus prägten Zusammenstöße mit der Polizei, Sachbeschädigungen und vereinzelt gar Plünderungen von Geschäften die Tage. Die Polizei ihrerseits trat enorm hart auf und setzte bisweilen Grundrechte außer Kraft (vgl. Haunss et al. 2017, Komitee für Grundrechte und Demokratie 2017).

Ich möchte den Versuch unternehmen, die Ereignisse zu verorten. Zwei Fragen leiten das Vorhaben: Erstens, inwiefern stehen die Geschehnisse um den G20-Gipfel für eine Zuspitzung von Konflikten und damit für eine Repolitisierung von Gesellschaft? Sowie zweitens, wie fordern die Protestereignisse staatliche Akteure heraus und inwiefern sind deren Reaktionen als autoritär zu bezeichnen? Insgesamt arbeite ich fünf Aspekte heraus, die als einzelne Phänomene nicht per se neu sind, in der Summe aber die 
G20-Tage zu einem wichtigen Ereignis machen: Erstens ist eine deutliche Polarisierung politischer Positionen auszumachen; zweitens markieren die Protesthandlungen in Hamburg einen deutlichen Bruch mit der bestehenden Ordnung, was als Ausweitung antagonistischer Praxen diskutiert wird; drittens hat die Polizei wiederholt Grundrechte suspendiert; viertens waren die Tage von einer Vielzahl polizeilicher Meldungen geprägt, die sich später als nicht haltbar erwiesen, dies wird als mediale Eskalation bezeichnet; und fünftens ist eine Militarisierung politischer Konflikte auszumachen.

Zusammengenommen stehen die fünf Aspekte für eine gesellschaftliche Entwicklung, dieich thesenhaftals,Ende der Postdemokratie beziehungsweise ,Rückkehr der Politik' verstehe. Mit dem Begriff Postdemokratie wird ein gesellschaftliches Gefüge beschrieben, in dem demokratische Aushandlungsmechanismen eingeschränkt werden, eine Refeudalisierung von Entscheidungsprozessen innerhalb formal demokratischer Staatsgefüge zu beobachten ist und Widerspruch gegen die bestehende Ordnung primär über die Behauptung der Alternativlosigkeit derselben und erst sekundär mittels Gewalt verunmöglicht wird. Wenngleich vieles, was Postdemokratie ausmacht, Gültigkeit behält, ist das starre Bild der Alternativlosigkeit, so meine These (vgl. Mullis 2017a), heute brüchig geworden und es scheint möglich, etablierte Ordnung grundlegend herauszufordern. Dies führt nicht zwingend zu gesellschaftlichem Wandel, was mitunter auf zunehmend autoritäre Handlungsweisen staatlicher Akteure zurückzuführen ist.

Grundlage des Argumentes bilden zwei ineinandergreifende Reflexionsebenen: zum einen der Rückgriff auf theoretische Vorarbeiten und zum anderen eine qualitative Auswertung von Presseberichten und Bewegungserzeugnissen.

Theoretische Eckpfeiler sind die Überlegungen von Jacques Rancière (1997; 2002 [1995]; 2008 [2000]) und dessen Konzeption von Politik und Unvernehmen sowie Giorgio Agambens (2004) Reflexionen zum Ausnahmezustand. Der Beitrag reiht sich ein, in eine - auch innerhalb der kritischen Stadtforschung - anhaltende Diskussion über die Potenziale postfundamentalistischer Philosophie[3], beziehungsweise deren Perspektiven auf Politik, für gesellschaftswissenschaftliche Fragestellungen.

In die qualitative Auswertung der Medienberichterstattung eingeflossen sind 174 Zeitungsartikel, die von März bis Ende Juli 2017 in Onlineportalen von überregionalen Tageszeitungen erschienen sind.[4] Das Hamburger Abendblatt (HA) dient als zentrale Quelle. In den Datenkorpus aufgenommen wurden Berichte von anderen Medien, sofern sie Ereignisse anders darstellen oder zuvor nicht beleuchtete Aspekte aufgreifen. Analysiert wurden Aufrufe zu den Aktionstagen sowie Bewertungen der Ereignisse von lokalen Akteuren. Die vorgenommene Auswahl des empirischen Materials wurde mittels MAXQDA induktiv kodiert. Ziel war eine Rekonstruktion der Ereignisse sowie eine Zusammenstellung der Erklärungsmuster und Deutungen. Es geht mir weder um eine Diskursanalyse, noch um eine Bewertung unterschiedlicher Positionen der Presseorgane. Die Auswertung zielt auf den Dialog zwischen den theoretischen (Vor-)Überlegungen und den empirischen Befunden.

Um die fünf Aspekte und die damit verbundene These des Endes der Postdemokratie auszuarbeiten, werde ich zunächst in die Debatte um Postdemokratie, Politik und Ausnahmezustand einführen und die These ein 
erstes Mal aufgreifen. Im dritten Kapitel werde ich die Protestereignisse in Hamburg knapp darstellen und darauf aufbauend im vierten Kapitel die fünf Aspekte diskutieren. Abschließend kehre ich zur leitenden These zurück.

\section{Postdemokratie/ Ausweitung des Ausnahmezustands}

In den letzten Jahren hat eine Debatte über den Zustand der demokratischen Institutionen und Funktionsweisen der Repräsentation eingesetzt (vgl. Merkel 2016). Diese Diskussionen stehen nicht zuletzt im Kontext der Diskussion um Neoliberalisierungsprozesse und deren Auswirkungen auf die Konstitution von Gesellschaft (vgl. Brenner/Theodore 2002, Butterwegge et al. 2008, Harvey 2005). ,Postdemokratie“ ist hierbei ein zentraler Begriff (vgl. Böhnke 2011, Michelsen/Walter 2013, Öztürk 2011, Schulze 2012, Swyngedouw 2009; 2011; 2013, Schümer 2012), der zumindest in zwei divergenten Weisen bestimmt wird (Mullis/Schipper 2013): Zum einen politikwissenschaftlich im Anschluss an Colin Crouch (2008), zum anderen im Rahmen der postfundamentalistischen Zuwendungen zu dem Politischen, wobei Jacques Rancière (1997) ein zentraler Ideengeber ist.

Crouch (2008: 13) argumentiert, dass die Verfahren und Institutionen, die Demokratie als Staatsform ausmachen, wie etwa freie Wahlen, ein Rechtsstaat oder Beteiligungsmöglichkeiten weiterhin intakt seien oder mittels Partizipationsverfahren gar gestärkt würden. Gleichzeitig diagnostiziert er einen wachsenden Einfluss von wirtschaftlichen Eliten, wodurch Demokratie refeudalisiere und an inkludierendem Gehalt verliere. Crouch (2008) fasst unter Postdemokratie Prozesse wie den Abbau des Wohlfahrtsstaates, sinkende Wahlbeteiligungen und Personalisierung von Politik, die Ausweitung von marktförmigem Verhalten, die Flexibilisierung der Arbeitswelt sowie eine Tendenz zur Ökonomisierung und Kommodifizierung gesellschaftlicher Güter.

Zeitdiagnostisch zieht Rancière (1997; 2002 [1995]; 2016 [2005]) ähnliche Schlüsse. Sein zentrales Argument ist hingegen auf einer allgemeineren Ebene verortet. So ist für ihn Postdemokratie primär ein über Herrschaft eingerichtetes gesellschaftliches Gefüge, das den Anspruch erfolgreich verteidigt objektiv und alternativlos zu sein. Eric Swyngedouw (2013: 148) schlussfolgert, dass Postdemokratie davon lebt, ,alle in eine konsensuelle pluralistische Ordnung einzubeziehen und/oder diejenigen, die sich außerhalb des Konsens stellen, radikal auszuschließen“. Weit wichtiger als die faktische Ausgestaltung von demokratischer Staatlichkeit ist für Rancière, dass eine gesellschaftliche Ordnung eingerichtet wird, in der Politik als Akt der Infragestellung von Ordnung erfolgreich unterminiert wird. Für Mark Davidson und Kurt Iveson (2015) bedeutet dies, dass weniger die Kategorie der Postdemokratie als vielmehr die Praxen ihrer Infragestellung von Interesse sein sollten. Dies führt zum Begriff der Politik.

Politik ist für Rancière (2014: 148) in Bezug auf einen konkreten Konflikt der antagonistische Kampf zwischen „zwei Wahrnehmungswelten“, die aufeinandertreffen und einen Streit über die Bestimmung des Umstrittenen eingehen (ebd.: 236). Unvernehmen sowie die Forderung nach Gleichheit bilden die Grundlage (Rancière 2002 [1995]: 12). Mit Unvernehmen benennt er eine Situation, in der beide Seiten sich zwar hören, unter dem Gesagten aber völlig Unterschiedliches verstehen (ebd.: 9f.). Die Forderung nach 
Gleichheit und der Versuch mittels Praxis das spaltende Unvernehmen zu überwinden, macht für ihn Politik aus. Sie stellt die in historischen Prozessen und herrschaftlich abgesicherte Ordnung, die reguliert, wer Anteil an der Gestaltung von Welt hat und wer nicht, in Frage. Politik ist eine Praxis, in der ein Kollektivsubjekt den „Anteil der Anteillosen“ (ebd.: 27) einfordert, den Antagonismus offenlegt und sich in einem Akt der Selbstermächtigung zu einer Welt zählt, zu der es nicht gehören soll. Damit steht Politik nicht für die Lösung des Streites um das Vernehmen, sondern für die Praxis, die den Antagonismus und den Raum des Dissens erst produziert (Rancière 2011: 10).

Hier komme ich nun ein erstes Mal auf die These des Endes der Postdemokratie zu sprechen. Folgen möchte ich den Ausführungen von Crouch und Rancière was diekonkrete Beschreibung von Gesellschaft angeht. Mit Rancières Politik aber möchte ich gegen seine Bestimmung von Postdemokratie argumentieren, denn das Mantra der Alternativlosigkeit ist heute nicht mehr durchsetzbar und damit die Logik der Postdemokratie zerbrochen. Zwei Indizien sind relevant: Einerseits eine zu beobachtende Polarisierung von Gesellschaft während der letzten zehn Jahre und andererseits, dass die weltweiten Revolten des Jahres 2011 - vom Arabischen Frühling, über die europäischen Bewegungen der Plätze bis hin zu Occupy - das handlungsmachtbezogene Selbstverständnis politischer Bewegungen verschoben haben.

Oliver Decker und Elmar Brähler (2016: 95) argumentieren, dass das letzte Jahrzehnt von politischen Auseinandersetzungen und Transformationen geprägt sowie von Polarisierungseffekten bestimmt war: Gerungen wurde um den Umgang mit der Finanz- und Wirtschaftskrise, der Politik der Austerität. Jüngst waren es - einmal mehr - die Auseinandersetzungen um Migration, Flucht und Zuwanderung, die die politischen Debatten prägten. Die deutsche Wirtschaft boomt, gleichzeitig aber führten die Reformen des Sozialstaates der 2000er zur Verstetigung von Armut (Butterwegge 2016). Aufstiegschancen stagnieren für viele, wenn sie nicht gar in einen Trend nach unten umschlagen, so Oliver Nachtwey (2016).

Aktuell ist es vor allem der Aufstieg rechtsextremer Parteien und Bewegungen, die die Debatten um Politisierung von Gesellschaft innerhalb kritischer Sozialwissenschaften prägen (vgl. Geiselberger 2017). Dabei gerät in Vergessenheit, dass sich, wie Donatella della Porta (2017: 67) es ausdrückt, aktuell auch die „progressive Seite“ sozialer Bewegungen „bester Gesundheit“erfreut. Erinnert sei an die weltweiten Revolten des Jahres 2011 (vgl. Birke/Henninger 2012, PROKLA 177), aber auch an die Gezi-Proteste in der Türkei 2013. In Deutschland hat Blockupy über Jahre hinweg Protest gegen die europäische Krisenpolitik organisiert (Mullis 2017b). Widerspruch kommt auch von vielfältigen Streikkampagnen bei Amazon, in Kitas oder im Pflegebereich. Nicht zu vergessen der Kampf gegen den BraunkohleAbbau und für Klimagerechtigkeit von „Ende Gelände“, die Mobilisierung von Migrant_innen für Bleibe- und Bewegungsrecht oder die vielfältigen „Recht auf Stadt“-Bewegungen. All diesen Bewegungen gelingt es mal mehr, mal weniger aus dem Unvernehmen hervorzutreten und Widerspruch hörbar zu machen, womit sie Alternativlosigkeit aufspalten und einen progressiven Pol in die Polarisierungen einführen.

Als zweites Indiz dafür, dass das Mantra der Alternativlosigkeit nicht mehr trägt, möchte ich die Verschiebung des handlungsmachtbezogenen 
Selbstverständnisses von politischen Bewegungen anführen. So war Protest auch in Zeiten des gesteigerten postdemokratischen Unvernehmens der 1990er und 2000er nie wirklich abgeebbt. Neu ist heute der Rahmen durch welchen Protestereignisse von Aktiven in den Bewegungen selbst betrachtet werden. Das Jahr 2011 hat tiefe Spuren hinterlassen. Mit den Ereignissen ging die Gewissheit einher, dass politische Kämpfe nicht allein appellieren, sondern im Hier und Jetzt in lokalisierten Auseinandersetzungen auch radikalen Wandel einleiten können (vgl. Azzellini 2014, Mullis 2017b).

Diese Transformation der Perspektive verändert Bewegungen und markiert im Vergleich zur globalisierungskritischen Bewegung mit deren Mobilisierungen nach Seattle 1999, Prag 2000, Genua 2001 oder Heiligendamm 2007 ein verändertes Selbstverständnis. Verändert hat sich weniger die Form der Proteste - Demonstrationen, ziviler Ungehorsam und riot sind geblieben - verschoben hat sich das gesellschaftliche Gefüge in und mit welchem progressive Bewegungen zu agieren haben. War der Slogan der 2000er ,Eine andere Welt ist möglich“ und die Bewegungen von einer Perspektive der transnationalen und globalen Kooperation geprägt (vgl. Daphi 2017), würde der Slogan heute wohl eher lauten: ,Eine andere Welt ist nötig'. Vor dem Hintergrund der erfahrenen Krise und der konkreten Angriffe auf das gesellschaftliche Gefüge liegt die Perspektive heute weit stärker in der Transformation der jeweiligen ,lokalen' Alltäglichkeiten, die in der Summe in eine andere Globalität münden kann. Ein abstraktes Gefühl der kapitalistischen Ungerechtigkeit war gerade im globalen Norden für viele der Antrieb sich an den globalisierungskritischen Bewegungen zu beteiligen. Heute sind es weit öfter konkrete Erfahrungen des Ausschlusses, die die Proteste der letzten Jahre in Europa bestimmten (vgl. Birke/Henninger 2012, Daphi 2017, Huke 2016, Mullis 2017b, Nachtwey 2016, PROKLA 177).

Zusammen verdeutlichen die beiden Punkte, dass Alternativen - wenn auch nicht nur progressive - zur bestehenden gesellschaftlichen Ordnung formuliert werden und gesellschaftlicher Wandel zur Möglichkeit wird. Damit wird Postdemokratie brüchig.

Zum Erhalt der Ordnung, so wird in der kritischen Europaforschung und den Politikwissenschaften argumentiert (vgl. Demirović 2013, Forschungsgruppe ,Staatsprojekt Europa' 2012, Kretschmann/Legnaro 2017, PROKLA-Redaktion 2016, Purtschert et al. 2008, Kannankulam 2008), greifen staatliche Organe immer öfter auf autoritäre Mittel zurück. Vielfach wird dabei die Persistenz und Durchsetzungskraft von Staatlichkeit betont. Mir scheint, dass dies zu kurz greift und dass autoritäre Mechanismen stärker in Relation zur Politisierung von Gesellschaft gesetzt werden müssten, womit Potenziale Ordnung aufzubrechen sichtbarer würden. Es ist der Blick ins „Krisenlabor Griechenland“ (Hartmann/Malamatinas 2011) und insbesondere der Blick auf die urbanen Krisenproteste in Athen, der hilft, eine solche Perspektive zu entwickeln (vgl. Crisis Scape 2014, Douzinas 2013, Koutrolikou 2016, Köse 2016, Mullis 2017b, Stavrides 2012, Vradis 2014). Mit Verweis auf den Ausnahmezustand nach Giorgio Agamben werden in den Analysen zu den Krisenprotesten in Athen Politik und deren Verunmöglichung mittels autoritärer Praktiken durch staatliche Organe relational in Beziehung gesetzt. Ausnahmezustand zielt darauf, Politik durch faktisches Aussetzen von Recht sowie mittels Produktion von Unsicherheit und Angst zu unterbinden. 
Für Agamben (2004: 33) stellt Ausnahmezustand „eine Reaktion auf unmittelbare, reale Gefahren“ dar, „zu deren Abwehr gesetzlich verbürgte Rechte für eine begrenzte Zeit aufgehoben werden“. Ausnahmezustand bedeutet nicht ein Regieren außerhalb des Rechtes, vielmehr markiert er einen Grenzfall:

„Technisch gesehen ist das Spezifische am Ausnahmezustand weniger die mangelnde Gewaltenteilung, auf der immer wieder insistiert wird, als dass die Gesetzeskraft aus dem Gesetz herausgelöst wird. Der Ausnahmezustand definiert einen Zustand des Gesetzes, in dem Norm zwar gilt, aber nicht angewandt wird (weil sie keine,Kraft' hat), und auf der anderen Seite Handlungen, die nicht den Stellenwert von Gesetzen haben, deren ,Kraft' gewinnen.“ (Agamben 2004: 49)

Mit Blick auf Rancières Politik markiert die Implementierung des Ausnahmezustands nichts anderes als den Moment, in dem die Alternativlosigkeit durch politische Praxis als nicht haltbar entlarvt und dies autoritär beantwortet wird. Zum Erhalt der brüchig gewordenen Ordnung setzen staatliche Akteure qua Reproduktion und Erhalt eben dieser Ordnung bestehendes Recht außer Kraft (Mullis 2017b: 200-203).

\section{Der G2o-Gipfel in Hamburg}

Im Folgenden werde ich das im vorhergehenden Kapitel allgemein formulierte Argument zum Ende der Postdemokratie anhand der Ereignisse um den G2O in Hamburg vertiefen. Zunächst stelle ich hier jedoch die Geschehnisse Anfang Juli 2017 kurz dar.

Im Februar 2016 kündete Bundeskanzlerin Angela Merkel (CDU) nach Absprachen mit Hamburgs Erstem Bürgermeister Olaf Scholz (SPD) an, das G20-Treffen in der Hansestadt durchzuführen. Für Hamburg sei der Gipfel „eine große Chance“ (Hamburg 2016) und Innensenator Andy Grote (SPD, zit. nach MoPo 14.5.2017) kündete ein „Festival der Demokratie“ an. Gewerkschafter_innen, Aktive der Partei Die Linke, NGOs sowie Gruppen der radikalen Linken begannen alsbald mit der Planung von Protesten. Die erwartete Anwesenheit von Recep Tayyip Erdoğan, Wladimir Putin oder Donald Trump, beziehungsweise die autoritäre Politik für die diese stünden, waren zentrale Themen der Aufrufe (vgl. BlockG2O 2017, G2O-Protestwelle 2017, Grenzenlose Solidarität statt G20! 2017, Jugend gegen G20 2017, Shutdown-Hamburg 2017, Welcome to Hell 2017).

In Hamburg wurden die Vorbereitungen für die Proteste dadurch befeuert, dass das Treffen in den an das Schanzenviertel anrainenden Messehallen abgehalten werden sollte: Befürchtet wurden drastische Beeinträchtigungen des öffentlichen Lebens. Im Rahmen zweier Stadtteilversammlungen von „St. Pauli selber machen“, eine im November 2016 und eine im Juni 2017, an der jeweils mehrere Hundert Menschen teilnahmen, wurde klar gegen die G20 Stellung bezogen und Proteste mit vorbereitet.[5]

Noch bevor die ersten Demonstrationen stattfanden, glich Hamburg einer Festung. Die „schlimmsten Krawalle“ stünden bevor, so die Polizeiführung (zit. nach NDR 26.3.2017). Über 20.00o Polizist_innen, rund 3.00o Einsatzfahrzeuge, mehrere Dutzend Wasserwerfer, über 20 Helikopter, Räumpanzer 
und Spezialeinheiten wurden zusammengezogen. Um die An- und Abreise der Gipfelteilnehmer_innen zu garantieren, wurde von der Innenstadt bis zum Flughafen eine 38 km2 große Demonstrationsverbots- und Sicherheitszone eingerichtet (HA 2017).

Zwischen dem 22. Juni und dem 9. Juli fanden über 130 Kundgebungen und Demonstrationen sowie unter dem Motto „Gipfel für globale Solidarität“[6] ein Gegengipfel statt (HA 14.7.2017). Während der Protesttage wurde die Innenstadt Hamburgs in einen kollektiven Protestraum verwandelt. Vielerorts hingen von Balkonen und in Schaufenstern Banner mit Slogans und Parolen. Mehr als 24 Orte öffneten ihre Tore für Aktivist_innen als Rückzugsund Erholungsraum.[7]

Die Lage eskalierte ein erstes Mal, als die Polizeiführung wider der gerichtlichen Verfügung am Sonntag den 2. Juli entschied (Justiz Hamburg 3.7.2017), den Aufbau des Schlafcamps im Elbpark Entenwerder mittels Einsatzhundertschaften zu unterbinden. Aufgrund der anhaltenden Proteste wurden Schlafcamps letztlich an anderen Stellen bewilligt. Auch am 4. Juli ging die Polizeiführung hart vor. Am Abend waren Tausende dem Aufruf zum cornern, zum ausgelassenen Zusammenkommen auf Hamburgs Straßen, gefolgt. Gegen 22 Uhr forderte die Polizei die Menge auf, die Kreuzung am Neuen Pferdemarkt zu räumen. Als diese den Anweisungen nicht nachkam, wurden Wasserwerfer eingesetzt (TAZ 5.7.2017). Die Anwesenden quittierten dieses Vorgehen mit dem Ruf: „Das ist unsere Stadt!“ (Recht auf Stadt PM 5.7.2017). In beiden Fällen wurde in der Presse Unverständnis über das polizeiliche Vorgehen geäußert. So hieß es im NDR nach dem verhinderten Campaufbau: „Deeskalation - schon mal gehört? Man reibt sich verwundert die Augen, was sich in Hamburg abspielt, noch bevor der G2O-Gipfel überhaupt losgeht. Kooperatives Konfliktmanagement der Polizei? Fehlanzeige!“ (NDR 3.7.2017). Noch am 4. Juli plädierte die Süddeutsche Zeitung dafür, dass auch „zorniger Protest“ erlaubt sein muss und die TAZ konstatierte nach dem Wasserwerfereinsatz am Neuen Pferdemarkt zweierlei Bewertungsrahmen:

„Man muss sich das mal vorstellen: Würde ein G20-Gipfel in, sagen wir, Sankt Petersburg stattfinden, und würden noch vor Beginn des Gipfels Menschen, die beim abendlichen Bier zusammen stehen, mit Wasserwerfern auseinandergetrieben - hierzulande wäre die Hölle los. Polizeistaat!, würde es heißen, Verletzung des Grundrechts auf Versammlungsfreiheit!“ (TAZ 5.7.2017)

Von der Polizei unbehelligt blieb indes eine Aktion von Recht auf Stadt-Aktivist_innen, bei der am 2. Juli die Grünanlage am Neuen Pferdemarkt besetzt und in „Arrivati Park“ umbenannt wurde (Arrivati Park 2.7.2017). Im Laufe der Woche wurde der Park zu einem Knotenpunkt verschiedener Gruppen und Initiativen. Verbunden wurde der Protest gegen die G2O mit Protesten gegen Gentrifizierung und urbane Exklusion sowie den Kämpfen von Illegalisierten um Bleiberecht (Recht auf Stadt PM 5.7.2017). Entstanden ist, so die Initiator_ innen, ein „Ort der Solidarität jenseits der Grenzen nationaler Zugehörigkeit“, der auch nach dem G2O als Ankerpunkt „für eine solidarische Stadt von morgen"Bestand haben solle (Recht auf Stadt PM 11.7.2017).

Für den Vorabend des Gipfelbeginns am 6. Juli war unter der Parole „Welcome to Hell“ die dritte große Demonstration angekündigt. Jeweils 
rund 20.000 Menschen hatten bereits am 2. Juli an der „Protestwelle“ sowie am 5. Juli an der Tanzdemo „Lieber tanz ich als G20“ teilgenommen. Nun waren es Gruppen aus dem links-autonomen Spektrum, die angekündigt hatten, den „größten schwarzen Block, den es je gab“ (zit. nach HA 1.7.2017) bilden zu wollen und der „Aufführung der Macht in Hamburg ganz praktisch entgegenzutreten und unsere Vorstellungen einer gerechteren und solidarischen Gesellschaft entgegenzusetzen“ (Welcome to Hell 2017). Während die Auftaktkundgebung nachmittags ohne Zwischenfälle verlief, eskalierte die Situation nach Beginn der Demonstration schnell. Rund 1.00o Menschen hatten an der Spitze der Demonstration einen Block gebildet und sich vermummt, was die Polizeiführung nicht hinnehmen wollte. In der engen Straße St. Pauli Fischmarkt stoppten Hundertschaften der Polizei die rund 15.00o Demonstrant_innen und forderten dazu auf, Vermummungsutensilien abzunehmen. Die Verhandlungen dauerten mehr als 45 Minuten und wie die Presse berichtete, folgten viele Teilnehmende der Aufforderung (HA 7.7.2017, NDR 7.7.2017). Noch während die Gespräche liefen, stürmten Hundertschaften der Polizei mit großer Härte in die Demonstration - ein Auslöser war nicht ersichtlich.[8] Panik brach aus und die Situation wurde unübersichtlich. Dutzende Kleingruppen zogen bis spät in die Nacht durch die Stadt. Seitens der Demonstrant_innen wurden Sachbeschädigungen verübt und es kam zu Zusammenstößen mit der Polizei, wobei auf beiden Seiten Menschen teils schwer verletzt wurden. Immer wieder sammelten sich große Demonstrationszüge (TAZ 7.7.2017a, Zeit 7.7.2017). Die Berliner Zeitung (BZ 7.7.2017) resümiert exemplarisch für den medialen Tenor (vgl. FR 6.7.2017a; b, NDR 7.7.2017, TAZ 7.7.2017a; b, Zeit 7.7.2017): Die militante Linke sei eindeutig verantwortlich für die Eskalation, jedoch auch die Polizei sei einem „Exzess von Sicherheitswahn“ verfallen, habe unnötig an der „Gewaltspirale“ gedreht, friedlichen Protest verunmöglicht und dabei die Kontrolle verloren.

Am darauffolgenden Morgen, dem 7. Juli, begann der G20-Gipfel. Tausende strömten in Richtung Sicherheitszone, um dort die Anreise der Gipfelgäste zu blockieren. Gleichzeitig versuchten Hunderte die Hafenanlagen zu erreichen, um die „Logistik des Kapitals“ (Shutdown-Hamburg 2017) lahmzulegen. Nachmittags demonstrierten tausende Schüler_innen im Rahmen von „Jugend gegen G20“ (2017) und rund um die Elbphilharmonie wurde versucht, den Fototermin der Gruppe der 20 zu stören. Über diese Aktionen des zivilen Ungehorsams hinaus wurden in den frühen Morgenstunden an mehreren Orten der Stadt schwere Sachbeschädigungen begangen und es kam zu Angriffen auf Polizeikräfte. Abends brachen im Schanzenviertel erneut schwere Auseinandersetzungen zwischen Aktivist_innen und der Polizei aus. Über mehrere Stunden hinweg brannten Barrikaden meterhoch, schwere Sachbeschädigungen wurden verübt und Geschäfte geplündert. Gemäß Darstellung der Polizeiführung (HA 9.7.2017), sahen sich die Einsatzkräfte außerstande der Lage Herr zu werden. Die Vehemenz der Angriffe sowie Hinterhalte, aufgrund derer die Polizeiführung mit Attacken auf Einsatzkräfte mit Molotowcocktails, Gehwegplatten, Eisenstangen, Zwillen und potenziell gar mittels Schusswaffen rechnete, habe ein Vorrücken ins Viertel verunmöglicht. Um die Ausschreitungen zu beenden, wurde letztlich der Einsatz von schwerbewaffneten Sondereinsatzkommandos (SEK) angeordnet (vgl. HA 10.7.2017a; 13.7.2017; 20.7.2017). 
Über den ganzen Tag hinweg ging die Polizei mit großer Härte vor. In den sozialen Medien kursierten Bilder von Wasserwerfereinsätzen gegen Sitzblockaden. Dokumentiert wurde der Einsatz von Pfefferspray aus nächster Nähe sowie Schlagstockhieb, Tritte und Faustschläge.[9] Für mediale Aufregung sorgte, dass am Eingang zum G20-Pressezentrum neun akkreditierten Journalist_innen der Zutritt verwehrt wurde - auf Polizeilisten kursierten 32 Namen (FR 12.7.2017) - und dass Journalist_innen und Anwält_innen erheblich an der Ausübung ihrer Arbeit gehindert wurden (vgl. Huffington Post 8.7.2017). Die Ereignisse am 7. Juli veränderten jedoch die Berichterstattung und die in den Tagen zuvor formulierte Kritik am Vorgehen der Polizei begann zu schwinden. Der vormals als legitim erachtete Protest wurde zunehmend als „Exzess“ beschrieben (HA 10.7.2017a).

Am nächsten Tag, Samstag den 8. Juli, stand zum Abschluss der Proteste die Demonstration „Grenzenlose Solidarität statt G20“ auf dem Programm. An der Demonstration, die weitgehend ohne Zwischenfälle verlief, nahmen über 75.00o Menschen teil. Es war die größte politische Versammlung in Hamburg seit den 1980er Jahren. In der Presse wurde sie kaum noch thematisiert.

\section{G20-Proteste: fünf Aspekte zu Politik und Ausnahmezustand}

Die in Hamburg jeweils konkreten politischen Artikulationen sowie die Implementierung des Ausnahmezustandes möchte ich nun ins Zentrum rücken. Ausgehend von den im zweiten Kapitel entwickelten Argumenten thematisiere ich fünf Aspekte, die für sich genommen nicht neu sind, in der Summe aber die G20-Tage zu einem wichtigen Ereignis machen. Die beiden Indizien für das Ende der Postdemokratie - Polarisierungen sowie das veränderte handlungsmachtbezogene Selbstverständnis politischer Bewegungen - führen zu den ersten beiden Aspekten: In Hamburg werden erstens vielschichtige Polarisierungseffekte zwischen politischen Positionen konkret und zweitens ist eine Ausweitung antagonistischer Praxen auszumachen. Ferner habe ich im zweiten Kapitel argumentiert, dass Ausnahmezustand und die Ausweitung autoritärer staatlicher Regierungsweisen mit dem Ende der Postdemokratie in Beziehung stehen. Ausnahmezustand kann zugleich Ausgangspunkt und Resultat von politischem Widerspruch sein. Auf jeden Fall ist er eng mit Versuchen verknüpft, eine spezifische Ordnung aufrecht zu erhalten. Für die Proteste gegen den G2o haben Daniel Loick (2017), Danijel Majic (2017) und Thomas Seibert (2017) Mechanismen des Ausnahmezustandes benannt. Um diese zu konkretisieren, möchte ich drei weitere Aspekte diskutieren: drittens, die Suspendierung von Grundrechten, was für das faktische Aussetzen von Recht steht. Sowie viertens die mediale Eskalation und fünftens die Militarisierung politischer Konflikte, die beide als spezifische Mechanismen der Produktion von Unsicherheit und Angst interpretiert werden.

\section{Polarisierung politischer Positionen}

Es sind zumindest drei Dimensionen, in denen Polarisierungseffekte zum Ausdruck kamen. Erstens erwiesen sich die G2O-Staaten als gespalten und sie hatten Mühe, ein geeintes Projekt zu formulieren. Donald Trump 
verabschiedet sich von Freihandel und Klimaschutz, mit Wladimir Putin bestehen erhebliche Differenzen bezüglich des Syrien- und Ukrainekonfliktes, Recep Tayyip Erdoğan forciert den autoritären Umbau der Türkei und seitens der europäischen Mitglieder scheint die einende Maxime gerade noch im Streben nach (ökonomischer) Stabilität und dem Schutz der EUAußengrenze vor Flüchtlingen zu liegen. Konsens bestand lediglich darin, den Kampf gegen den Terror voranzutreiben (Spiegel 9.7.2017c).

Zweitens sind die vielfältigen und umfassenden Proteste als Anzeichen für politische Polarisierungseffekte zu nennen. In den diversen Aufrufen finden globale und nationale Polarisierungen Widerhall (vgl. BlockG20 2017, G2OProtestwelle 2017, Grenzenlose Solidarität statt G20! 2017, Jugend gegen G2O 2017, Shutdown-Hamburg 2017, Welcome to Hell 2017). Es dominiert die Einschätzung mit den bisherigen Protesten gegen die Austerität nicht erfolgreich gewesen zu sein und gleichzeitig zuschauen zu müssen, wie die Weltordnung brüchig wird.

Die Polarisierung manifestiert sich drittens in der Repräsentation der Ereignisse. Vor allem im Nachhinein klafften die Bewertungen weit auseinander. In der medialen Aufarbeitung sowie in den Bewertungen von offizieller Seite dominieren die Ereignisse des Freitagabends im Schanzenviertel, während seitens lokaler Gruppen versucht wird, ein breiteres Bild der Ereignisse zu zeichnen und kritische Fragen zu stellen.

„Wir haben eine neue Qualität von Gewalt erlebt“ stellt Heiko Maas (zit. nach FR 10.7.2017b) fest. Für Bundesinnenminister Thomas de Maizière (zit. nach FR 10.7.2017a) war klar: Die „Randalierer“ sind „verachtenswerte, gewalttätige Extremisten, genauso wie Neonazis und islamistische Terroristen“. CDU-Präsidiumsmitglied Jens Spahn (zit. nach FR 11.7.2017) sprach von „Linksfaschisten“, der damalige SPD-Parteivorsitzende Martin Schulz (zit. nach TAZ 10.7.2017a) von „Mordbrennern“. Der außenpolitische Sprecher der SPD-Fraktion Niels Annen (zit. nach HA 8.7.2017a) verglich die Situation mit „Afghanistan und anderen Krisenregionen“. Im Hamburger Abendblatt ist die Rede von „Bildern wie aus dem Bürgerkrieg“(HA 7.7.2017; 10.7.2017a). Die Forderungen nach härteren Strafen und mehr Repression überschlugen sich. Der Staat habe jahrelang vor der linken Gefahr die Augen verschlossen und dies müsse nun ein Ende nehmen (vgl. FAZ 13.7.2017, SZ 10.7.2017, TAZ 10.7.2017b). Gefordert wurden Meldeauflagen, Fußfesseln und die Einführung einer europaweiten Extremismusdatenbank (vgl. SZ 10.7.2017, TAZ 10.7.2017b). Ins Zentrum der Kritik rückten in den Tagen nach den Protesten autonome Zentren in fast allen größeren deutschen Städten - allen voran die „Rote Flora“ in Hamburg. Deren Umfeld wurde als Drahtzieherin der Ausschreitungen ausgemacht, weshalb Konsequenzen folgen müssten (vgl. TAZ 10.7.2017b).

Aus Hamburg kam jedoch auch deutlicher Widerspruch zur dominanten Darstellung der Ereignisse in der Presse und durch politische Repräsentant_innen (vgl. Centro Sociale PM 19.7.2017, Geschäftstreibende aus dem Schanzenviertel 12.7.2017, Recht auf Stadt PM 11.7.2017). So beschreibt Andreas Diederich (zit. nach FR 18.7.2017b), der am Schulterblatt ein Geschäft betreibt, die Stimmung im Viertel als vergiftet. Er relativiert aber das Ausmaß der Schäden, die, abgesehen von vereinzelten Plünderungen, nicht schlimmer seien als nach einem normalen 1. Mai: „Das macht es nicht 
besser. Aber es ist ganz sicher nicht so, dass der Stadtteil in Trümmern liegt.“ (ebd.). Die Wut über die Krawallmacher_innen sei spürbar, jedoch bestünde auch viel Unverständnis über das Agieren der Polizei und den tagelangen Belagerungszustand. Ähnliches beschreiben einige Geschäftstreibende aus dem Schanzenviertel (12.7.2017), die die „Komplexität der Dynamik, die sich in dieser Nacht hier Bahn gebrochen hat [...] weder in den Medien noch bei der Polizei oder im öffentlichen Diskurs angemessen reflektiert" sehen.

Um den sehr unterschiedlichen Wahrnehmungen und Erfahrungen einen Raum zu geben, organisierte „St. Pauli selber machen“ in Zusammenarbeit mit weiteren Initiativen am 20. Juli eine außerordentliche Stadtteilversammlung. 1.200 Menschen kamen. Die Luft sei zum Schneiden und die geäußerten Positionen kritisch bis kontrovers gewesen. Trotzdem sei die Gesprächsatmosphäre von „gegenseitigem Respekt, Rücksichtnahme und einer großen Aufmerksamkeit“ geprägt gewesen (St. Pauli selber machen 26.7.2017, vgl. auch 12.10.2017). Gemeinsam verabschiedeten die Anwesenden per Akklamation ein Statement. Dieses lässt die unterschiedlichen Erfahrungen im Stadtteil gleichberechtigt stehen, weist aber auch die Instrumentalisierung lokaler Erfahrungen zurück, um damit ein schärferes Vorgehen gegen linke Projekte und Demonstrationen zu begründen:

„1. Wir wollten keinen G2O und jetzt wissen wir noch besser, warum. 2. Wir haben unsere eigenen Erfahrungen. Wir lassen uns nicht diktieren, was passiert ist. Wir werden uns weiter austauschen und mitteilen. 3. Rote Flora und alle anderen linken Zentren bleiben!“

(St. Pauli selber machen 26.7.2017)

Kontroversen um die Deutung von Ereignissen gehören zu Protesten (vgl. Mullis 2017b: 227-272). Nach den G2O-Tagen verrutschten die Maßstäbe jedoch in Gänze und die zweifelsohne dramatischen Ereignisse der Freitagnacht wurden zum alleinigen Signifikanten. Vergessen war alles zuvor Geschehene, die vielfältigen Aktionen sowie das harsche Vorgehen der Polizei. Pointiert argumentiert die TAZ(10.7.2017a): Das Handeln Weniger in einem sehr isolierten Bereich Hamburgs sei zwar „völlig idiotisch, brutal, überflüssig" gewesen, legitimiere die Reaktion auf die Proteste aber in keiner Weise.

Mit Verweis auf Rancières Politik verstehe ich dieses Ringen um Deutung als integralen Bestandteil politischer Auseinandersetzungen. Mittels der drastischen Verengung des Narrativs auf den Gewaltausbruch vom Freitagabend durch Behörden, politische Repräsentant_innen und viele Medien, wurde versucht, den aufgebrochenen Widerspruch zurück ins Unvernehmen zu drängen. Auf der anderen Seite wird auf eine breitere Betrachtung gepocht, was ich als Kampf um die Fähigkeit sich politisch artikulieren zu können, interpretiere.

\section{Ausweitung antagonistischer Praxen}

Als zweites Indiz für das Ende der Postdemokratie habe ich oben für eine Verschiebung des handlungsmachtbezogenen Selbstverständnisses politischer Bewegungen argumentiert. Dies wird in der Ausweitung antagonistischer Praxen während des G2o deutlich. Hierfür stehen die starke 
Fokussierung auf Praxen des zivilen Ungehorsams sowie die Logik des Aufstandes, die den Freitagabend im Schanzenviertel prägten.

Aktionen des zivilen Ungehorsams haben Tradition in Deutschland (Burschel et al. 2014). Angestoßen von den globalen Protesten 2011 wurden sie im Rahmen von Blockupy in Frankfurt aber wesentlich ausgeweitet (vgl. Mullis 2017b: 280-282). Die Erfahrungen von 2011 sorgten für ein verändertes Vertrauen in die eigenen Handlungsmöglichkeiten und die Finanz- und Schuldenkrise vermittelte das Gefühl, dass es nötig sei, den Bruch mit der Ordnung jetzt zu vertiefen. Hinsichtlich der legitimierenden Narrative wurde ziviler Ungehorsam als Praxis in Stellung gebracht, um selbstbestimmt, radikal aber gewaltfrei den Bruch mit der gesellschaftlichen Ordnung zu markieren. So bewerteten im Zuge der G2O-Proteste bei einer Befragung von Demonstrant_innen am 8. Juli - also nach den schweren Zusammenstößen 81,4 Prozent zivilen Ungehorsam als legitime und angemessene Protestform, die nicht als Gewalt zu verstehen sei (Haunss et al. 2017: 22).

Es geht darum den Bruch mit der herrschenden Ordnung zu markieren; eine Stoßrichtung, die auch im Aufruf zu den Aktionen von „BlockG20“ deutlich wird:

„Diese G20 repräsentieren uns nicht! Sie haben keine Lösungen für die realen Probleme unserer Welt. Ihr Wirtschaftssystem ist ein einziger Wahnsinn: ohne Respekt vor der Zukunft, der Natur und dem Recht aller Menschen auf ein gutes Leben in Würde und Freiheit. Sollen wir zuschauen, wie sie unsere Welt zugrunde richten? [...] Wir setzen auf den rebellischen Verstand und die Vernunft des Herzens. Wir kündigen an: Wir werden die Regeln überschreiten und die Rote Zone dicht machen.“ (BlockG20 2017)

Praxen des Ungehorsams prägten die Proteste in Hamburg auch dort, wo nicht explizit dazu aufgerufen wurde und die Proteste entwickelten eine starke Eigendynamik: Die Menschen gingen nicht nach Hause als die Errichtung des Camps in Frage gestellt wurde. Ähnliches gilt für die Aktionen des cornerns. Der Konflikt um das Recht sich zu versammeln wurde sichtbar. Trotz des harten Vorgehens der Polizei drangen am Tag des Gipfelbeginns Tausende in die Demonstrationsverbotszone vor und versuchten die Anreise der Gipfelgäste zu stören. Im Arrivati Park harrten die Menschen aus, auch als die Ausschreitungen tobten. Ein Aspekt des Ungehorsams ist auch bei den 75.000 Menschen auszumachen, die an der Demonstration „Grenzenlose Solidarität statt G20“ teilnahmen: Sie machten sich trotz der Bilder des Freitagabends, als SEK-Einheiten schwer bewaffnet im vom Krawall gezeichneten Schanzenviertel aufmarschierten und eine gespenstige Stimmung einer eskalierten urbanen Unruhe verbreiteten, auf den Weg in die Hansestadt.

In diesem Kontext fasse ich im Anschluss an Robin Celikates (2010) zivilen Ungehorsam als eine Praxis der Politik. Ausschlaggebend ist für Celikates, dass ziviler Ungehorsam das Potenzial entfaltet, Bühnen des Dissenses zu errichten. Es werde nicht nur symbolisch in Gesellschaft eingegriffen, sondern die Bedingungen von gesellschaftlicher Ordnung selbst über ein absichtliches, rechtswidriges und prinzipienbasiertes kollektives Protesthandeln in Frage gestellt (ebd.: 280). Es handele sich also um eine politische Praxis, die 
Antagonismen explizit macht und Alternativlosigkeit negiert. Die Legitimität der Praxis entspringt, so Celikates, nicht aus einem staatlichen Recht, sondern aus dem damit entwickelten demokratischen Potenzial.

In Hamburg kam die Ausweitung antagonistischer Praxen aber nicht nur im zivilen Ungehorsam zum Ausdruck. Es ist ferner die Logik des Aufstandes, die ich hier diskutieren möchte. Diverse Kommentator_innen haben die Ereignisse im Schanzenviertel am Freitagabend mit den urbanen riots in Paris 2005 oder London 2011 verglichen und diese als unpolitische und kriminelle Akte gerahmt (vgl. HA 8.7.2017a; b; 10.7.2017a; 12.7.2017, Spiegel 9.7.2017b, TAZ 9.7.2017; 14.7.2017, Zeit 10.7.2017). In den letzten Jahren wurden urbane riots wissenschaftlich verstärkt diskutiert. Thematisiert wurden Wut und Empörung über Polizeigewalt als Auslöser, Gewalt und Sachbeschädigung als Artikulationsmuster und die meist marginalisierten und exkludierten Subjekte der Ereignisse. Nicht zuletzt wurde, entgegen der Entpolitisierungsthese, der politische Gehalt der Ereignisse herausgestellt (vgl. Altenried 2012, Dikeç 2017, Frenzel et al. 2016, Mayer et al. 2016; für Hamburg: Mücke/Rinn 2016). In Bezug auf Praxen und Ausdruck der Ereignisse sind für Hamburg Parallelen zu den Diskussionen um riots zu erkennen. Mit Blick auf das veränderte handlungsmachtbezogene Selbstverständnis wird jedoch ein Aspekt relevant, der in den Diskussionen nicht angesprochen wird: die Logik des Aufstandes als bewusste, politische Strategie der Markierung des Antagonismus.

Prominent wurde die Logik des Aufstandes vom anonym schreibenden „Unsichtbaren Komitee“ (2010 [2007]) formuliert. Das Manifest der Gruppe „Der kommende Aufstand“ ist ein pathetischer Aufruf zur Subversion und zum Entzug aus der Ordnung. Aufstand wird als Mittel beschrieben, um den Antagonismus mit der bestehenden Ordnung zu artikulieren. In gewisser Weise macht die Logik des Aufstandes den riot an sich sprechfähig, darüber hinaus wird auf die Formulierung einer konkreten Forderung aber verzichtet.

„Nicht sich sichtbar machen, sondern die Anonymität, in die wir verbannt wurden, zu unseren Gunsten wenden und durch die Verschwörung, durch die nächtliche oder maskierte Aktion aus ihr eine unangreifbare Angriffsposition machen. Der Brand vom November 2005 ist ihr Modell. Keine Anführer, keine Forderung, keine Organisation, aber Worte, Gesten, Komplizenschaft.“ (Unsichtbares Komitee 2010 [2007]: 91f.)

Am Abend des 7. Juli wurde im Schanzenviertel ein solcher Aufstand inszeniert. Die Ereignisse folgten einer - wenn auch nicht in Verlautbarungen verbalisierten - Logik: jener des unmittelbaren Bruchs und der Zurückweisung von Ordnung in und durch direkte Aktion. Darüber, ob Aufstand an sich und die darin aufblitzende Neuformierung von Welt ausreicht, um eine Alternative zur bestehenden Ordnung zu formulieren, muss jedoch gestritten werden. Zumal sich mit Blick auf Rancières Konzeption von Politik die Frage aufdrängt, wie in der reinen Negation das Potenzial einer Affirmation, die zwingender Bestandteil der Errichtung gesellschaftlicher Ordnung - auch einer egalitären - ist (Mullis 2017b: 47-70), gefunden werden soll.

Insgesamt sprechen die beiden Aspekte - Polarisierung und Ausweitung antagonistischer Praxen - so kontrovers sie auch sein mögen, für eine 
gesteigerte Konflikthaftigkeit von Gesellschaft und die Vertiefung politischer Auseinandersetzungen. Lange stabil geglaubte Ordnungen werden in Frage gestellt und herausgefordert. ,Postdemokratie wurde von Rancière als Begriff bestimmt, um eine Situation zu beschreiben, in der Politisierung unterminiert wird und der Logik der Alternativlosigkeit folgt. Heute erleben wir das Ende dieses Zustandes.

\section{Suspendierung von Grundrechten}

Die Suspendierung von Grundrechten war in Hamburg allgegenwärtig, so der Anwaltliche Notdienst beim Republikanischen Anwältinnen- und Anwälteverein (RAV PM 9.7.2017): Ein „Festival der Grundrechtsverletzungen“ sei es gewesen. „Massenhaft wurde das Recht auf kollektive Meinungsäußerungsfreiheit, auf Versammlungsfreiheit, auf körperliche Unversehrtheit und auf Achtung der Menschenwürde beschränkt.“ (ebd.) Zwei Beispiele stehen hierfür: das Vorgehen gegen das Camp im Entenwerder Elbpark sowie der Anmeldebescheid für die Demonstration „Welcome to Hell“. Im ersten Fall wurde Recht seitens der Polizei faktisch außer Kraft gesetzt, im zweiten einzig der Form nach eingehalten.

Nach wochenlangem Ringen um die Errichtung von Zeltstädten als Unterkunft für Aktivist_innen wurden diese schließlich verboten. Innensenator Grote (zit. nach HA 1.7.2017) begründete dies damit, dass gewaltbereite Aktivist_innen im Camp „Unterschlupf finden“ könnten, womit „sie zu einem Rückgrat der militanten Infrastruktur" würden. Das Hamburger Verwaltungsgericht setzte am 1. Juli das Verbot wegen mangelnder Begründung außer Kraft (Justiz Hamburg 3.7.2017). Als die Aktivist_innen am 2. Juli das Camp im Entenwerder Elbpark aufzubauen begannen, setze sich die Polizeiführung eigenmächtig über das Urteil hinweg und ging gegen den Aufbau vor. Im NDR (3.7.2017) wird dies als Versuch interpretiert „Infrastruktur der G2O-Gegner lahmzulegen“, was einem „verfassungswidrigen Grundrechtseingriff" gleichkomme.

Im Rahmen der Demonstration „Welcome to Hell“ war es die Anmeldebestätigung, die überraschte. Die Demonstration war ohne Auflagen mit einer Route bis zum Messegelände bewilligt worden. Gleichzeitig wurden seitens der Polizei heftige Ausschreitungen prognostiziert (MoPo 5.7.2017). Der Anwaltliche Notdienst (PM 5.7.2017) zeigte sich irritiert: Üblicherweise würden solche Demonstrationen „mit telefonbuchdicken Auflagenkatalogen“ konfrontiert. Der Subtext sei klar, ,ihr werdet den Kundgebungsplatz nie erreichen“ (ebd.). Dem formalen Ablauf war zwar Genüge getan, die damit einhergehenden Rechte wurden jedoch im Rahmen des polizeilichen Vorgehens bei der Auflösung der Demonstration komplett ausgehöhlt. So kommen die Vorgänge, nach den Worten des Rechtsanwaltes Udo Vetter in der TAZ (7.7.2017b), einem „Dolchstoß für das Grundgesetz“ gleich.

Suspendierung von Recht beschränkte sich in den Tagen nicht auf diese Beispiele. Vermeldet wurde eine ganze Reihe unbegründeter Massenfestnahmen (FAZ 31.7.2017); die flächendeckende Überwachung des Mobilfunknetzes (TAZ 31.7.2017); und die Behinderung von Anwält_innen, Sanitäter_innen sowie Journalist_innen (vgl. Komitee für Grundrechte und Demokratie 2017, Riot Medics Berlin PM 17.7.2017). In einem Gastbeitrag für 
die Frankfurter Rundschau skizziert Daniel Loick (2017) die Implikationen des polizeilichen Vorgehens: Im Rechtsstaat würden der Polizei umfassende Kompetenzen und das Gewaltmonopol eingeräumt. Legitimität gewinne Polizei dadurch, dass in einer Demokratie die Staatsgewalt vom Volke ausgehe und die Institution Polizei dessen Wille lediglich exekutiere. Dies funktioniere aber nur so lange, wie Grundrechte in vollem Umfang gewahrt würden. Beginne Polizei diese eigenmächtig zu beschneiden, stelle sie den „Wesensgehalt der liberalen Demokratie“ in Frage, Polizei werde zur politischen Akteurin und polizeiliches Handeln verwandle sich in „schiere Repression“.

\section{Mediale Eskalation}

Für den Aspekt der medialen Eskalation, den ich als Teil der Produktion von Unsicherheit und Angst diskutieren möchte, können zwei Beispiele angefügt werden: die Polizeikommunikation und die Debatte um Polizeigewalt.

Zur Rolle der Polizei fällt auf, mit welch eskalierender Rhetorik deren Leitung agierte. In den Tagen der Proteste veröffentlichte sie eine ganze Reihe von dramatischen Meldungen über schwere Angriffe, Plünderungen oder Verletzungen und begründete damit ihr Vorgehen. Immer wieder sah sich die Polizeiführung jedoch genötigt solche Meldungen zu dementieren (Tagesschau 10.7.2017). Besonders schwer wiegt hierbei die polizeiliche Kommunikation am Abend der schweren Ausschreitungen im Schanzenviertel. Die Polizeiführung hatte mehrfach betont, dass schwere Angriffe und von Autonomen errichtete Hinterhalte ein Vorrücken von Einsatzkräften verunmöglichen würden. In den Tagen danach mehrten sich kritische Rückfragen in den Medien und immer lauter wurden Beweise gefordert. Als diese auch mehrere Tage nach den Ereignissen noch immer nicht vorgelegt worden waren, wurden die Zweifel an der polizeilichen Darstellung immer lauter (vgl. MoPo 13.7.2017, NDR 19.7.2017, SZ 19.7.2017). Im Oktober musste die Hamburger Innenbehörde letztlich einräumen, dass sie weder für Hinterhalte noch für schwere Bewaffnung Beweise gefunden habe (FR 24.10.2017) - eine intensive mediale Aufarbeitung dieses Eingeständnisses blieb indes aus.

Seitens der Aktivist_innen und Protestbeobachter_innen waren die Polizeigewalt und die Einschränkungen der Presse- und Anwaltsarbeit die zentralen Themen (vgl. Centro Sociale PM 19.7.2017, Geschäftstreibende aus dem Schanzenviertel 12.7.2017, RAV PM 8.7.2017; 9.7.2017; 14.7.2017, Riot Medics Berlin PM 17.7.2017 / auch www.g20-doku.org, letzter Zugriff am 28.11.2017 / in den Medien FAZ 17.7.2017, MoPo 17.7.2017, ND 12.7.2017, 16.7.2017, Spiegel 26.7.2017, Tagesschau 21.7.2017, TAZ 13.7.2017). Kontrovers wurde die Diskussion, als Hamburgs Bürgermeister Olaf Scholz (zit. nach HA 9.7.2017) die „heldenhafte Tätigkeit“ der Beamt_innen lobte und an anderer Stelle ausführte: „Polizeigewalt hat es nicht gegeben“ (zit. nach Spiegel 14.7.2017).

Der Sprecher des Hamburger Senates (HA 17.7.2017) sowie Andy Grote (FR 19.7.2017a) sahen sich angesichts 35 bereits Mitte Juli laufender Verfahren gegen Beamt_innen sowie dokumentierter Übergriffe genötigt, die Aussage zu relativieren. Seitens der Behörden wurde jedoch betont (HA 17.7.2017), dass es Ursache und Wirkung nicht zu verdrehen gelte und bei über 20.000 schwer belasteten Beamt_innen die Vorkommnisselediglich 
Einzelfälle seien. Die Zahl der Verfahren gegen Polizist_innen stieg. Im März 2018 wurde in 135 Fällen ermittelt, davon in 108 wegen Körperverletzung im Amt (NDR 16.3.18).

Deutlich wird an den beiden Beispielen zum einen, dass einmal produzierte Narrative und Bilder sich nicht mehr grundlegend verschieben lassen und zum anderen, dass Polizei und Stadtverwaltung in keiner Weise als neutrale oder zumindest beruhigende Akteur_innen auftraten. Gerade die Polizeiführung hat intensiv interveniert und mit ihrer Kommunikation eine Stimmung der Gefahr und Angst miterschaffen, was als Strategie der Delegitimierung von Protest sowie des Unvernehmen-Machens zu beurteilen ist. In diesem Sinne ist auch die Internetfahndung zu bewerten, wobei die Hamburger Polizei mittels Fotofahndung nach mutmaßlichen Täter_innen öffentlichkeitswirksam sucht.[10]

\section{Militarisierung politischer Konflikte}

Zuletzt möchte ich nun noch auf die Militarisierung politischer Konflikte eingehen. Es handelt sich dabei um einen zentralen Mechanismus, der die Delegitimierung von Protest in gewisser Weise verräumlicht. Nach Stephen Graham (2010) wurden Städte in den letzten Jahren immer mehr zu Orten der Unsicherheit und Gefahr stilisiert, die seitens Sicherheitsstrateg_innen vor allem wegen der schwierigen Ausübung von Kontrolle Aufmerksamkeit erfahren. Ereignisse wie Gipfelproteste werden in diesem Gefüge, so Graham (2014: 40), zu „Schaufenstern“ für den new military urbanism, der militärische Strategien und Ausrüstung in zivile Konflikte überträgt und anwendbar macht:

„Das Problem bei der neuen Ausrichtung des new military urbanism liegt darin, dass demokratische Bemühungen und Bewegungen der Zivilbevölkerung zu der gleichen Sorte von Bedrohung gezählt werden wie LKW-Bomben oder Selbstmordattentate im al-Qaida-Stil. Militarisierte Polizeieinheiten gehen überaus einschüchternd gegen Menschen vor, die von ihren demokratischen Bürgerrechten Gebrauch machen." (Graham 2014: 40, Herv. i. O.)

Auch für Deutschland macht Rafael Behr (2015) eine Tendenz aus, Terror und Protest als militärische Bedrohungen zu fassen, womit eine stete Aufrüstung der Polizei begründet wird. Die Geschehnisse um den G20 Gipfel erachte ich als zentrales Ereignis in dieser Entwicklung.

Seitens der Hamburger Polizei wird bereitwillig eingeräumt, dass „die massive Polizeipräsenz auch einen Abschreckungseffekt" entfachen sollte (HA 2017). Das Vorgehen gegen Protestierende mit Wasserwerfern, Schlagstöcken und Pfefferspray passt in dieses Gefüge. Aktivist_innen, Anwält_innen und Sanitäter_innen betonen einhellig, dass die Polizeigewalt allgegenwärtig war und es sich keineswegs um Einzelfälle handelte. Selbst im Spiegel (9.7.2017a) wird die Verallgemeinerung von behördlicher Gewalt thematisiert: „Wenn man Polizisten das Gefühl gibt, der Rechtsstaat sei optional, verhalten sie sich auch so." In der Gesamtschau der Ereignisse und in Relation zu ähnlichen Einsätzen in Deutschland, entsteht der Eindruck, dass die Null-Toleranz der Hamburger Linie (vgl. Teune 2017) die Übergriffe unmittelbar implizierte, wenn sie nicht gar zur Raison des Einsatzes gehörten. 
Der Einsatz des SEK stellt eine besondere Eskalation dar. Am Abend des 7. Juli rückten schwer bewaffnete Einheiten mit Schussfreigabe ins Schanzenviertel vor (HA 13.7.2017). Die produzierten Bilder sind gespenstig. Wirklich nötig war der Einsatz - nach heutigem Wissensstand - wohl nicht und so dürfte das Kalkül auch in der Produktion von Bildern der Härte und des Durchgreifens gelegen haben.

Durch die militärische Rhetorik, die den Einsatz begleitete, wird diese Wahrnehmung noch verstärkt: Da schreibt ein Polizist (zit. nach HA 12.7.2017), „wir haben bis an unsere Belastungsgrenzen und darüber hinaus gekämpft“. Zum kämpfenden Polizisten passen die vielfach bemühten „bürgerkriegsähnlichen Zustände“ (HA 8.7.2017a). Ein Mitglied des SEK (zit. nach HA 13.7.2017) schreibt, dass er seinem Gefühl nach nicht „gegen Demonstranten“, sondern gegen „Verbrecher“ vorgegangen sei, „die versucht haben, sowohl Polizeibeamte als auch die Bevölkerung an Leib und Leben zu schädigen“. Der bereits zuvor zitierte Polizeibeamte (zit. nach HA 12.7.2017) mutmaßt, dass der „Tod von Polizisten und Bürgern anscheinend gewünscht“ war. Es ist die Rede von polizeilichen „Aufklärungskräften“ und von „effektiver“ autonomer „Gegenaufklärung“ (NDR 19.7.2017), die in der „Schlacht auf der Schanze“ (Zeit 8.7.2017) zum Einsatz gekommen sei. Seitens der radikalen Linken seien Kampfzeichen und Kommandostrukturen zu beobachten gewesen. Hergestellt wird ein Bild, in dem die Metapher des Krieges wahrgemacht wird und damit auch die militärische Antwort legitimiert.

Bedeutsam für die Diskussion des Ausnahmezustandes ist, so möchte ich mit Stavros Stavrides (2012) argumentieren, dass dessen Produktion erst über seine Verräumlichung voll zum Tragen kommt. Über die Bilder der Sicherheitskräfte sowie auch die mediale Inszenierung der brennenden Barrikaden im Schanzenviertel - egal wie räumlich isoliert die Ereignisse wirklich waren (WOZ 8.7.2017) - wird ein Raum der Unsicherheit geschaffen, eine Zone außer Kontrolle, in der besondere Maßnahmen legitim sind. In der kriegerischen Rhetorik werden sie verallgemeinert und vermittelt: ,Protest ist gefährlich, bleibt besser zu Hause‘. Das gefährliche Andere findet eine unmittelbare räumliche Verankerung und kann beobachtet werden, genauso wie auch das harte Vorgehen gegen sie zur Schau gestellt wird. Ausnahmezustand findet in der militärischen Kontrolle von Raum seine konkrete Materialisierung und Erfahrbarkeit.

Die Dimension des Polizeieinsatzes fügt sich in das Bild der letzten Jahre, wonach politische Konflikte sicherheitspolitisch bearbeitet werden sollen: Der Umgang mit den Krisenprotesten aber auch die jüngste Debatte um Flucht und Migration, die seit der Kölner Silvesternacht 2015/2016 im Lichte der inneren Sicherheit geführt wird, erzählen diese Geschichte. Dazu haben die islamistischen Anschläge der letzten Jahre auch in Deutschland die Präsenz von schwerbewaffneten Einheiten im alltäglichen Stadtgefüge normalisiert. So erstaunt nicht, dass Rufe nach vermehrten Einsätzen des SEK gegen Demonstrationen laut werden und Hamburg kein Einzelfall bleiben soll (vgl. TAZ 13.7.2017). Und dies auch nicht geblieben ist, wie der Einsatz des SEK anlässlich einer Demonstration von Antifaschist_innen in Wurzen, Sachsen zeigt (MDR 4.9.2017).

Anwendung von Zwang und dessen Legitimierung sind aber selbst Teil gesellschaftlicher Auseinandersetzungen (vgl. Mullis et al. 2016) und so 
bedarf die Implementierung des Ausnahmezustandes zumindest der partiellen Zustimmung. Das Ausbleiben einer klaren Kritik des polizeilichen Vorgehens in Hamburg zeigt, dass das Aussetzen von Grundrechten und Gewalt zur Unterbindung von Protest durch Polizei von demokratischen Politiker_innen als legitim erachtet wird. Es ist ein Beleg für den Befund von Decker et al. (2016), dass harte Strafen für „Unruhestifter“ eine wachsende Zustimmung finden (ebd.: 55f.).

Deutlich wurdein Hamburg, dass dieMechanismen des Ausnahmezustandes der Delegitimierung des Protestes sowie der Abschreckung dienen sollten. Es galt politische Praxen zu unterbinden, oder sie zumindest ins Unvernehmen zu drängen. Gewalt seitens der Polizei sowie deren Negation sind gleichermaßen Teil der auf Angst und Unsicherheit setzenden Rationalität, wie Falschmeldungen, die das Bild einer kriegerischen Eskalation befeuerten. Die Implementierung des Ausnahmezustands verstehe ich somit als zur Politik inverse Markierung des Endes der Postdemokratie.

\section{Das Ende der Postdemokratie?}

Es ist stets eine Herausforderung und bisweilen ein unbefriedigendes Unterfangen, eine auf einer hohen Abstraktionsebene verortete These, wie hier das ,Ende der Postdemokratie‘ beziehungsweise die ,Rückkehr der Politik‘, im Rahmen konkreter kleinteiliger Auseinandersetzungen zu diskutieren. Stets steht die Argumentation unter Verdacht, dem Ansinnen der allgemeinen Abstraktionsebenen auf der Ebene des Konkreten nicht gerecht zu werden. Dennoch gilt: Wenn die abstrakt beschriebenen Prozesse und Tendenzen auf der Ebene des Konkreten nicht zumindest einen Widerhall finden, funktioniert die abstrakte Beschreibung nicht. Ich habe somit versucht die leitende These in den Auseinandersetzungen um den G2O-Gipfel in Hamburg zu konkretisieren. Zwei Fragen waren hierfür relevant: Inwiefern findet Politik während der G2O-Tage eine Zuspitzung und wie haben die Protestereignisse staatliche Akteure herausgefordert beziehungsweise inwiefern sind deren Reaktionen mit der Kategorie des Ausnahmezustandes beschreibbar?

Eine deutliche Ausweitung politischer Praxen ist festzustellen und die bestehende Ordnung wurde durch die Aktivist_innen, aber auch innerhalb der G20 selbst, herausgefordert. Ziviler Ungehorsam ist ein bedeutsames Mittel der Artikulation von Politik. Die staatlichen Autoritäten reagierten mit großer Härte auf die Proteste, suspendierten Grundrechte und reagierten mittels der Produktion von Angst und Unsicherheit auf die artikulierte Politik. Insgesamt lese ich die Ereignisse in Hamburg als Beleg für die formulierte These des Endes der Postdemokratie.

Mir ist wichtig, dass die These des Endes der Postdemokratie nicht missverstanden wird. Es geht mir nicht darum zu behaupten, dass herrschaftlich abgesicherte gesellschaftliche Strukturen per se flexibler werden. Das Ringen um das Vernehmen, das Einfordern des „Anteils der Anteillosen“ (Rancière 2002 [1995]: 27) wird zwar deutlicher, eine progressive Transformation von Gesellschaft ist daraus aber nicht zwingend abzuleiten. Vorerst bedeutet das Ende der Postdemokratie lediglich eine Multiplikation von Kämpfen und von gesellschaftlichen Bruchlinien. Unvernehmen-Machen, wozu die Implementierung des Ausnahmezustands einen wichtigen Beitrag 
leistet, ist ein bedeutsames Mittel der Stabilisierung von Ordnung. Agambens Konzeption hilft zu verstehen, so scheint mir, wie es sein kann, dass Ordnung trotz der Zunahme von politischer Bewegung einigermaßen stabil bleibt. Diese sollte jedoch nicht als reines top-down-Prinzip interpretiert werden. Die Ereignisse in Hamburg verdeutlichen, dass die Ausweitung autoritärer Praxen zur Unterbindung von progressiver Politik in breiten Teilen von Gesellschaft Anerkennung findet. Diese zugesprochene Anerkennung ist selbst aber Produkt von politischen Kämpfen um Deutung. Kurzum, schon die Wahrnehmung und Deutung des Ausnahmezustandes ist Teil des politischen Ringens.

Ein Anliegen des Textes war letztlich auch einen Beitrag zur Debatte um die Potenziale einer postfundamentalistischen Perspektive auf Politik für gesellschaftswissenschaftliche Fragen zu leisten. Zentral scheint mir, dass Rancières Politik und Unvernehmen sowie Agambens Ausnahmezustand wichtige Begriffe darstellen, um gesellschaftliche Entwicklungen konkret zu fassen. Insbesondere erlauben sie Bewegung, Kämpfe und politische Praxen ins Zentrum zu stellen, statt immer wieder die Starrheit der bestehenden gesellschaftlichen Ordnung zu betonen und diese damit zu reproduzieren.

\section{Endnoten}

[1] Mein Dank für kritische Rückmeldungen und Anmerkungen gebührt Priska Daphi, Joscha Metzger, Annina Mullis, Conny Petzold und Ronja Stiep sowie den beiden anonymen Gutachter_innen, die konstruktiv zur Überarbeitung beigetragen haben. Auch den Herausgeber_innen der s u b \u r b a n möchte ich für das Feedback sowie die investierte Arbeit danken. Die Veröffentlichung dieses Artikels wurde gefördert durch den Open-Access-Publikationsfonds der Leibniz-Gemeinschaft.

[2] Das Gremium der zwanzig wichtigsten Industrie- und Schwellenländer trifft sich seit 1999 regelmäßig. Im Zuge der Finanz- und Wirtschaftskrise 2007/o8 wurde das Treffen 2008 erstmals auf der Ebene der Staats- und Regierungschef_innen durchgeführt. In der Verwaltung der schwersten Wirtschaftskrise seit dem zweiten Weltkrieg nahmen die G20 eine bedeutsame Rolle ein. In Zeiten staatlicher Rettungsprogramme für Banken, antizyklischer Konjunkturpakete und Selbstzweifel leiteten sie 2009 die Rückkehr zur neoliberalen Programmatik von Wettbewerb und freien Märkten ein (G20 2009).

[3] Postfundamentalistische Philosophien betonen die Abwesenheit letzter Gründungsnarrative für gesellschaftliche Realitäten und verweisen darauf, dass jede Form der Objektivität ein Produkt von politischer Praxis und gesellschaftlicher Gründungsprozesse ist. Jede Ordnung ist also zumindest potenziell stets umkämpft, auf jeden Fall aber Produkt von historischen Kämpfen. Der postfundamentalistische Blick erlaubt gleichermaßen die Wirkmächtigkeit von Strukturen, Normen und Materialität herauszustellen, sie aber stets im Gefüge gesellschaftlicher Kämpfe zu verorten. Argumentiert wird für ein Primat des Politischen vor einer jeden Ordnung (vgl. Marchart 2010; 2013).

[4] Berücksichtigt wurden Texte von: Frankfurter Allgemeine Zeitung (FAZ), Frankfurter Rundschau (FR), Hamburger Abendblatt (HA), Hamburger Morgenpost (MoPo), Neues Deutschland (ND), Spiegel Online (Spiegel), Süddeutsche Zeitung (SZ), Die Tageszeitung (TAZ), Die Wochenzeitung (WOZ) sowie Die Zeit (Zeit).

[5] vgl. www.st-pauli-selber-machen.de (letzter Zugriff am 29.11.2017).

[6] vgl. www.solidarity-summit.org (letzter Zugriff am 20.11.2017).

[7] vgl. www.centrosociale.de/ZentrenG20 (letzter Zugriff am 11.8.2017).

[8] Die Ereignisse sind im Livestream des Hamburger Abendblattes vom 6.7.2017 ab Minute 55:30 nachzuvollziehen: https://youtu.be/HNUD9Wrzd6w?t=55m30s (letzter Zugriff am 9.4.2018). 
[9] vgl. www.g20-doku.org (letzter Zugriff am 28.11.2017)

[10] www.polizei.hamburg/g20-fahndungen (letzter Zugriff am 11.1.2018).

\section{Autor_innen}

Daniel Mullis ist Humangeograph. Seine Interessen liegen in der politischen Philosophie, Sozialprotesten sowie Krisendynamiken, aktuell insb. im Neuen Autoritarismus. mullis@hsfk.de

Die Publikation dieses Beitrags wurde durch den Open-Access-Publikationsfonds der LeibnizGemeinschaft ermöglicht.

\section{Literatur}

Agamben, Giorgio (2004): Ausnahmezustand. Frankfurt a.M.: Suhrkamp.

Altenried, Moritz (2012): Aufstände, Rassismus und die Krise des Kapitalismus. Münster: Edition Assemblage.

Azzellini, Dario (2014): Ein Epochenbruch. Die neuen globalen Proteste zwischen Organisation und Bewegung, in: Prokla 44/4, 495-512.

Behr, Rafael (2015): Angst 2.o, sozusagen, in: Die Zeit, 18.12.2015.

Birke, Peter / Henninger, Max (Hg.) (2012): Krisen Proteste. Berlin/Hamburg: Assoziation A.

Böhnke, Petra (2011): Ungleiche Verteilung politischer und zivilgesellschaftlicher Partizipation, in: APuZ 2011 1-2, 18-26.

Brenner, Neil / Theodore, Nikolas (Hg.) (2002): Spaces of Neoliberalism. Malden: Blackwell.

Burschel, Friedrich / Kahrs, Andreas / Steinert, Lea (Hg.) (2014): Ungehorsam! Disobedience! Theorie \& Praxis kollektiver Regelverstöße, Münster: edition assemblage.

Butterwegge, Christoph (2016): Armut in einem reichen Land, 4. Aufl., Frankfurt a.M.: Campus.

Butterwegge, Christoph / Ptak, Ralf / Lösch, Bettina (Hg.) (2008): Neoliberalismus, Wiesbaden: VS.

Celikates, Robin (2010): Ziviler Ungehorsam und radikale Demokratie. Konstituierende vs. konstruierte Macht? in: Bedorf, Thomas / Kurt Röttgers (Hg.), Das Politische und die Politik, Berlin: Suhrkamp, 274-300.

Crisis Scape (2014): Future Suspended. Film. www.crisis-scape.net (letzter Zugriff am 14.10.2014).

Crouch, Colin (2008): Postdemokratie. Frankfurt a.M.: Suhrkamp.

Daphi, Priska (2017): Becoming a Movement. Identity, Narrative and Memory in the European Global Justice Movement, London/New York: Rowman \& Littlefield International.

Davidson, Mark / Iveson, Kurt (2015): Recovering the politics of the city: From the ,post-political city to a ,method of equality ' for critical urban geography, in: Progress in Human Geography 39/5, 543-559.

Decker, Oliver / Kiess, Johannes / Brähler, Elmar (Hg.) (2016): Die enthemmte Mitte. Autoritäre und rechtsextreme Einstellung in Deutschland. Gießen: Psychosozial-Verlag.

Decker, Oliver / Brähler, Elmar (2016): Ein Jahrzehnt der Politisierung: Gesellschaftliche Polarisierung und gewaltvolle Radikalisierung in Deutschland zwischen 2006 und 2016, in: Decker, Oliver / Johannes Kiess / Elmar Brähler (Hg.), Die enthemmte Mitte. Autoritäre und rechtsextreme Einstellung in Deutschland. Gießen: Psychosozial-Verlag

della Porta, Donatella (2017): Progressive und regressive Politik im späten Neoliberalismus, in: Geiselberger, Heinrich (Hg.) (2017): Die große Regression, Berlin: Suhrkamp, 57-76.

Demirović, Alex (2013): Multiple Krise, autoritäre Demokratie und radikaldemokratische Erneuerung, in: Prokla 43/2, 193-215.

Dikeç, Mustafa (2017): Urban Rage. The Revolt of the Excluded, New Haven/London: Yale University Press.

Douzinas, Costas (2013): Philosophy and Resistance in the Crisis. Cambridge/Malden: Polity.

Forschungsgruppe ,Staatsprojekt Europa' (Hg.) (2012): Die EU in der Krise. Zwischen autoritärem Etatismus und europäischem Frühling, Münster: Westfälisches Dampfboot. 
Frenzel, Janna / Greif, Philippe / Klein, Fabian / Uhlmann, Sarah (2016): Riots - Zur Verortung eines unscharfen Phänomens, in: sub\urban 4/1, 7-24.

Geiselberger, Heinrich (Hg.) (2017): Die große Regression. Berlin: Suhrkamp

Graham, Stephen (2010): Cities under Siege. The New Military Urbanism, London/ Brooklyn: Verso.

Graham, Stephen (2014): Belagerte Städte. Die Militarisierung des Urbanen, in: dérive 57, 37-45.

Hartmann, Detlef / Malamatinas, John (Hg.) (2011): Krisenlabor Griechenland. Finanzmärkte, Kämpfe und die Neuordnung Europas. Berlin: Assoziation A.

Harvey, David (2005): A Brief History of Neoliberalism, Oxford: Oxford University Press.

Haunss, Sebastian / Daphi, Priska / Gauditz, Leslie / Knopp, Philipp / Micus, Matthias / Scharf, Philipp / Schmidt, Stephanie / Sommer, Moritz / Teune, Simon / Thurn, Roman / Ullrich, Peter / Zajak, Sabrina (2017): \#NoG2o. Ergebnisse der Befragung von Demonstrierenden und der Beobachtung des Polizeieinsatzes, Berlin: ipb working papers.

Huke, Nikolai (2016): Krisenproteste in Spanien. Zwischen Selbstorganisation und Überfall auf die Institutionen, Münster: edition assemblage.

Kannankulam, John (2008): Autoritärer Etatismus im Neoliberalismus. Zur Staatstheorie von Nicos Poulantzas. Hamburg: VSA.

Komitee für Grundrechte und Demokratie (2017): Geschichte der Eskalation eines einwöchigen Protestgeschehens. Demonstrationsbeobachtung des Komitees für Grundrechte und Demokratie vom 2. bis 8. Juli 2017 in Hamburg zum G20. Köln.

Koutrolikou, Penny (2016): Governmentalities of urban crises in inner-city Athens, Greece, in: Antipode 48/1, 172-192.

Köse, Dilan (2016): „Wir sind der Zorn“ (Exoume thn Orgh). Die Romanos-Bewegung 2014 in Griechenland als konstituierender Bruch im postpolitischen Krisenregime, in: sub $\backslash$ urban $4 / 1,53-74$.

Kretschmann, Andrea / Legnaro, Aldo (2017): Ausnahmezustände. Zur Soziologie einer Gesellschaftsverfassung, in: Prokla 47/3, 471-486.

Loick, Daniel (2017): Die Polizei als Risiko für die Demokratie, in: Frankfurter Rundschau, 28.7.2017.

Majic, Danijel (2017): Wann der Rechtsstaat wirklich gefährdet ist, in: Frankfurter Rundschau, 18.7.2017.

Marchart, Oliver (2010): Die politische Differenz, Berlin: Suhrkamp.

Marchart, Oliver (2013): Das unmögliche Objekt. Eine postfundamentalistische Theorie der Gesellschaft, Berlin: Suhrkamp.

Mayer, Margit / Thörn, Catharina / Thörn, Håkan (Hg.) (2016): Urban Uprisings. Challenging Neoliberal Urbanism in Europe, London: Palgrave.

Merkel, Wolfgang (2016): Krise der Demokratie? Anmerkungen zu einem schwierigen Begriff, in: APuZ 40-42, 4-11.

Michelsen, Danny / Walter, Franz (2013): Unpolitische Demokratie. Zur Krise der Repräsentation, Berlin: Suhrkamp.

Mullis, Daniel (2017a): Das Ende der Postdemokratie, den Pessimismus überwinden, in: PROKLA 47/3, 487-493.

Mullis, Daniel (2017b): Krisenproteste in Athen und Frankfurt. Raumproduktionen der Politik zwischen Hegemonie und Moment. Münster: Westfälisches Dampfboot.

Mullis, Daniel / Schipper, Sebastian (2013): Die postdemokratische und postpolitische Stadt in der Geschichte der kommunalen Selbstverwaltung, in: sub \urban 1/2, 79-100.

Mullis, Daniel / Belina, Bernd / Petzold, Tino / Pohl, Lucas / Schipper, Sebastian (2016): Social protest and its policing in the „heart of the European crisis regime“. The case of Blockupy in Frankfurt, Germany, in: Political Geography 55, 50-59.

Mücke, Julika / Rinn, Moritz (2016): Keine riots in Deutschland? Die Ereignisse in HamburgAltona im Sommer 2013, in: sub \urban 4/1, 111-130.

Nachtwey, Oliver (2016): Die Abstiegsgesellschaft. Berlin: Suhrkamp.

Öztürk, Asiye (2011): Postdemokratie? in: APuZ 1-2/2011, 2.

PROKLA 177 (2014): Globale Proteste zwischen Organisation und Bewegung

PROKLA-Redaktion (2016): Der globale Kapitalismus im Ausnahmezustand, in: PROKLA 46/4, 507-542.

Purtschert, Patricia / Meye, Katrin / Winter, Yves (Hg.) (2008): Gouvernementalität und Sicherheit. Zeitdiagnostische Beiträge im Anschluss an Foucault. Bielefeld: transcript. 
Rancière, Jacques (1997): Demokratie und Postdemokratie, in: Rado Riha (Hg.): Politik der Wahrheit. Wien: Turia + Kant, 94-122.

Rancière, Jacques (2002 [1995]): Das Unvernehmen. Frankfurt a.M.: Suhrkamp.

Rancière, Jacques (2008 [2000]): Zehn Thesen zur Politik. Berlin: Diaphanes.

Rancière, Jacques (2011): Moments politiques. Interventionen 1977-2009. Zürich: Diaphanes.

Rancière, Jacques (2014): Erfindung des Möglichen. Interviews 2006-2009. Wien: Passagen. Rancière, Jacques (2016 [2005]): Der Hass der Demokratie, Köln: August.

Schulze, Ingo (2012): Kapitalismus braucht keine Demokratie. www.sueddeutsche.de (letzter Zugriff am 28.11.2017).

Schümer, Dirk (2012): Postdemokratie. Europa schafft sich ab. www.faz.net (letzter Zugriff am 28.11.2017).

Seibert, Thomas (2017): Der Anteil der Anteillosen, in: Die Tageszeitung, 12.7.2017.

Stavrides, Stavros (2012): Emancipating Spatial Practices in Struggle Against the Urban „State of Exception“, Paper presented at the „International Conference: Spatialities of Exception, Violence and Memory“, 2012, Madrid.

Swyngedouw, Erik (2009): The antinomies of the postpolitical city: In search of a democratic politics of environmental production, in: IJURR 33/3, 601-620.

Swyngedouw, Erik (2011): Interrogating post-democratization: Reclaiming egalitarian political spaces, in: Political Geography 30, 370-380.

Swyngedouw, Erik (2013): Die postpolitische Stadt, in: s u b \u r b a n 1/2, 141-158.

Teune, Simon (2017): Das Scheitern der „Hamburger Linie“, in: Blätter für deutsche und internationale Politik 8/2017, 9-12.

Unsichtbares Komitee (2010 [2007]): Der kommende Aufstand. Hamburg: Nautilus.

Vradis, Antonis (2014): Crisis-scapes suspended, in: City 18/4-5, 498-501.

\section{G2o in Hamburg: Politics, Disagreement, State of Exception and the end of Post-Democracy}

The G2O summit took place in Hamburg on July 7th and 8th 2017. During this period, diverse mass protests were organized throughout the city, partly escalating to violence. In this article I develop five aspects, which as such are not new but in sum stand for an intensification of political conflicts and mark the G2O days as an important event: (1) polarization of political positions, (2) extension of antagonistic practices, (3) suspension of fundamental rights, (4) production offear through media strategies and (5) the militarization of political conflicts. In order to achieve that, the article on the one hand draws on Jacques Rancière's theoretical work on politics and disagreement, as well as Giorgio Agamben's theorizations on the "state of emergency". On the other hand, it is based on a qualitative evaluation of the media coverage, protest calls and evaluations of the outcome around the G2O. Against this background, I argue that the events in Hamburg indicate an end of post-democracy. 